\title{
Influence of Host and Geographical Regions on the Rumen Methanogens Diversity in Indian Cattle and Buffaloes
}

\section{Shraddha Trivedi}

National Institute of Animal Nutrition and Physiology

Pradeep Kumar Malik ( $\square$ malikndri@gmail.com )

National Institute of Animal Nutrition and Physiology https://orcid.org/0000-0002-6581-4772

Atul Purushottam Kolte

National Institute of Animal Nutrition and Physiology

Govindasamy Thirumalaisamy

National Institute of Animal Nutrition and Physiology

Vanitha Shyamili Kumar

National Institute of Animal Nutrition and Physiology

Sejian Veerasamy

National Institute of Animal Nutrition and Physiology

Arindam Dhali

National Institute of Animal Nutrition and Physiology

Raghavendra Bhatta

National Institute of Animal Nutrition and Physiology

\section{Research}

Keywords: Archaea, Buffaloes, Cattle, Geographical location, Methanogen, Methanobrevibacter, Methanomassiliicoccales, 16S rRNA

Posted Date: August 6th, 2020

DOl: https://doi.org/10.21203/rs.3.rs-53542/v1

License: (c) (i) This work is licensed under a Creative Commons Attribution 4.0 International License. Read Full License 


\section{Abstract}

Background Archaea belong to the domain Euryarchaeota, represents only 3-5\% of the rumen microbiota; however, they play a central role in maintaining the $\mathrm{H}_{2}$ pressure. Host species in conjunction with geographical regions may lead to the variable distribution of rumen methanogens. Indian cattle and buffaloes represent 13 and $53 \%$ of the global population, respectively. However, the influence of host species (bovine) and geographical regions on the methanogens distribution has not been investigated in Indian subcontinent. Therefore, the present study was undertaken to explore the rumen methanogens diversity in the cattle and buffaloes native to the two distinct geographical regions of India.

Results Next generation sequencing of 16S rRNA gene from 36 rumen fluid samples corresponding to cattle and buffaloes revealed that Methanobrevibacter (Mbr.) genus constituted largest fraction (55-62\%) of the rumen archaeal community. Furthermore, Methanobrevibacter gottschalkii was the most prominent methanogens with the significantly higher abundance in cattle. Methanomassiliicoccales represented $<10 \%$ of the total archaeal community and was found to be the most variable between these two host species. In north region, the distribution of Methanomassiliicoccales, such as Methanosphaera sp., Group 4, Group 8, Group 12 sp. and Group 9 sp. was significantly higher in cattle. The distribution of prominent methanogens, such as Mbr. gottschalkii and Mbr. ruminantium did not differ between two host species in the north region. However, Mbr. gottschalkii was significantly higher in buffaloes as compared to cattle in south region. Mbr. wolinii was exclusively detected in the cattle. Inter-region comparison established that Methanomassiliicoccales were the most variable between the two regions.

Conclusions Host species did not affect the distribution of hydrogenotrophic methanogens except for Mbr. gottschalkii. Methylotrophic methanogens, in spite of their limited representation, were most influenced by the host species and geographical regions. Overall, the Methanomassiliicoccales distribution was higher in cattle, and the environmental conditions in north region were conducive for their higher distribution. The variable distribution of methanogens indicated that host and geographical region oriented strategies needs to be developed for the reduction of enteric methane emission. Further studies are warranted to explore the impact of diet on the distribution of rumen methanogens between the host species within and across different environmental conditions.

\section{Background}

After $\mathrm{CO}_{2}$, methane is the second most significant greenhouse gas [1] present in the atmosphere at a concentration of about $1830 \mathrm{ppb}$ and increasing with an average of $10 \mathrm{ppb}$ per year [2]. Agriculture sector annually contributes $188 \mathrm{Tg}$ to the anthropogenic methane emission. Enteric fermentation is one of the largest sources of methane emission among the agriculture sector; wherein global livestock emits 80-90 Tg methane per year. Worldwide the respective contribution of cattle and buffaloes to the annual enteric methane emission is estimated 72.42 and $11.05 \mathrm{Tg}$. A recent report [3] from the country estimated annual enteric methane emission from the Indian cattle and buffaloes as 4.92 and $2.91 \mathrm{Tg}$, respectively. Thus, these two host species remained major emitters aggregately contributing $33.1 \%$ of the global enteric 
methane emission. In spite of representing one of the production inefficiencies, methanogenesis is generally referred as a wasteful but necessary process in the rumen. Methanogenesis act as a sink for fermentative $\mathrm{H}_{2}$ produced in the rumen. Rumen harbours millions of anaerobic microbes, which accomplish the feed fermentation and aid in removing waste products. Archaea belong to the domain Euryarchaeota represents only $3-5 \%$ of the rumen microbiota $[4,5]$; nevertheless, their role in maintaining the $\mathrm{H}_{2}$ pressure low within the desirable limits through methanogenesis is incredible [6, 7]. Unlike bacteria, archaea lack peptidoglycan in their cell wall [8]. A unique characteristic of methanogens is the presence of coenzyme $F_{420}$ [9], which mediates redox transformations essential for the catabolic pathways [10]. Rumen methanogen community is complex and their substrate requirement is different as majority of them utilize carbon dioxide and formate; while others use methylated compounds for producing methane. Till date, about 155 species of the methanogens associated to six orders and twenty-nine genera have been isolated from different ecosystems [11]. However, only seven species of methanogens have been isolated so far from the rumen ecosystem [12]. It has been reported that the isolated methanogens from the rumen represents $<10 \%$ of the archaeal community [13]. Understanding of the rumen microbiome particularly of archaea is critical in order to develop effective strategies for enteric methane amelioration. Recent culture-independent studies have confirmed that the rumen methanogenic community is complex and a large fraction of it has not been cultured yet and thus remains unidentified [14, 15]. Recent metagenomics approaches enabled rapid community analysis without introducing the cultivation bias or primers associated variations $[16,17]$. Recent next generation sequencing techniques certainly improved our understanding about the diversification and the metabolic capacity of the methanogenic archaea in the rumen [18-20].

Cattle and buffaloes with a corresponding number of 195 and 110 million, aggregately constitute more than half of the total livestock population in the country. India alone harbours about $13 \%$ and $53 \%$ of the world's cattle and buffalo population, respectively [21]. Cattle and buffaloes are accounted for $85 \%$ of the annual enteric methane emission in India [3]. A recent comparison among distantly located geographical regions revealed that livestock in north and south region respectively contribute to about 37 and $17 \%$ of the enteric methane emission [3]. Host species in conjunction with environmental conditions and distinct feeding practices could be accountable for the variation in the regional enteric methane emission from the livestock. Effect of host on the rumen microbiota establishment has been reported [22] and previous reports suggest an impact on the archaeal community composition [23-26]. Further, the body size of the host species also influences the abundance of rumen microbiota [27]. As buffaloes are generally heavier than the cattle in India, a variable archaeal community structure between cattle and buffaloes may exist. Moreover, the environmental conditions prevailing in different geographical regions influence the rumen archaeal community composition [28]. Two distinct geographical regions considered in the present study experience variable climatic conditions as north India encounters a cool and dry weather in winter and hot in summer; while relatively high temperature \& humidity are the common features of south India weather. Present study was undertaken aiming to compare the rumen archaeal community structure between cattle and buffaloes belonging to the two distinct north and south geographical regions of the country. We hypothesized that the rumen archaeal community structure is controlled by the host species and 
geographical locations. Although cattle and buffaloes significantly contribute to the food basket of animal origin and methane emission; nevertheless, there is no existing report, where the rumen archaeal community structure between these two important bovines considering the impact of distinct geographical locations has been investigated in Indian context.

\section{Methods}

\section{Collection of rumen fluid samples}

A total 36 rumen fluid samples were collected from the cattle $(N=19)$ and buffaloes $(N=17)$ native to two distinct geographical region of India. Uttar Pradesh, Punjab and Gujarat represented the north region; while south region was represented by the Karnataka and Tamil Nadu states (Fig. 1). About $50 \mathrm{~mL}$ of the rumen fluid samples were collected from the individual cattle and buffalo in each region. The samples were filtered through double layer of muslin cloth and placed in a box containing dry ice. Thereafter, the rumen fluid samples were transported to the laboratory and processed for the genomic DNA isolation.

\section{Dna Isolation}

To allow the sedimentation of dissolved micro feed particles, the rumen fluid samples were initially centrifuged (Kubota 6500, Japan) at $1500 \mathrm{rpm}$ for five minutes. The supernatant obtained from the short spinning of rumen fluid samples was then transferred to another tube. About $2 \mathrm{~mL}$ supernatant was taken into an Eppendorf tube and centrifuged at $13400 \mathrm{rpm}$ for 10 minutes at $4{ }^{\circ} \mathrm{C}$. A thick pellet obtained by the centrifugation was retained while the supernatant fraction was carefully removed. Subsequently, $1 \mathrm{~mL}$ lysis buffer was added, and then pellet was dissolved through gentle pipetting. The mixed content was transferred to a $2 \mathrm{~mL}$ sterile screw cap tube containing $0.5 \mathrm{~g}(0.1 \mathrm{~mm})$ pre-sterilized zirconia beads (Biospec USA). Repeat bead beating plus column method was followed for genomic DNA isolation in the present study [29]. The QIAamp DNA mini kit (Qiagen, Germany) was used as per the manufacturer's instructions. Quality of genomic DNA was checked using $0.8 \%$ agarose gel electrophoresis; while the concentration of DNA was confirmed with Qubit 4.0 (Invitrogen). Genomic DNA was used for generating $2 \times 300$ bp amplicons using Illumina MiSeq platform.

\section{Library Construction And Sequencing Of 16s Rrna Gene}

Amplicon libraries were prepared using Nextra XT kit (Illumina Inc.). Archaea specific primers Arch-344F 5'ACGGGGYGCAGCAGGCGCGA 3' (Wemheuer et al., 2012) and Arch-806R 5'GGACTACVSGGGTATCTAAT 3' (Takai and Horikoshi, 2000) were used for the amplification of V3-V5 regions of 16S rRNA gene. Illumina i 5 and $i 7$ adapters were added to the primers for generating the amplicons. Amplicon libraries were purified by AMPureXP beads (Beckman Coulter Life Sciences, USA) and analyzed on 4200 Tape Station system (Agilent Technologies, USA) using D1000 Screen Tape station. Approximately 10-20 pM of each library was loaded onto the MiSeq platform for cluster generation and sequencing. 


\section{Bioinformatics Analysis}

Raw paired end reads were imported to the QIIME2 [30] for analysis. The reads were processed using the q2-dada2 plugin. The DADA2 pipeline was used for detecting and correcting the amplicon sequence data, joining of paired end reads and removal of chimeric sequences. Thereafter, quality filtered non-chimeric sequences were clustered into amplicon sequence variants (ASVs) with $100 \%$ similarity. Representative sequences were classified using RIM-DB archaea specific database [31] with q2-feature-classifier plugin. Multiple sequence alignment and masking of the conserved regions of the representative sequences was performed using q2-alignment plugin. The filtered alignments were used for the construction of phylogenetic tree using q2-phylogeny plugin. The plugin q2-diversity was used for alpha and beta diversity estimates. Alpha diversity measures, such as Shannon index, Pielou's evenness, and Beta diversity indices, such as Bray-Curtis and UniFrac distances were calculated using the rooted phylogenetic tree and a sampling depth corresponding to the lowest number of sequences in any sample. The significance of the alpha diversity was estimated using the Kruskal-Wallis statistical test, whereas, beta-diversity significance was determined using the Permutational multivariate analysis of variance (PERMANOVA). Further, the OTU table was imported into MicrobiomeAnalyst [32] for differential abundance analysis. Sequences with low abundance were filtered out using default parameters (minimum count of 4 in at least $20 \%$ samples and interquartile range of 10\%). Differential abundance was performed using metagenomeSeq [33] with zero-inflated Gaussian mixture model (fitZig), and a $P$ value $<0.05$ was considered statistically significant. The co-occurrence analysis was performed using the CoNet 1.1.1 tool in Cytoscape 3.7.0 [34]. The correlations with a Fisher's Z score $P$ value threshold of 0.05 were considered as statistically significant. Heatmap of the significant taxa at genus level was constructed using ClustVis tool [35]. Core microbiome analysis was performed using microbiome V1.4.2 package [36] with 50\% minimum prevalence for different geographical regions at genus and species level.

\section{Results}

A total of 11.3 million paired end reads were generated from 36 rumen fluid DNA samples collected from cattle $(N=19)$ and buffaloes $(N=17)$ native to two distinct geographical regions of India. The corresponding paired end reads in cattle and buffaloes were 6.99 and 4.31 million, respectively. Following the stringent quality filtering, about 1.55 million sequences (Table 1) were retrieved from both the cattle ( 0.8 million) and buffaloes ( 0.74 million). The sample wise filtering details and number of sequences retained at each step are provided in Additional file 1, Table S1. Rarefaction curve for Shannon index and observed OTUs confirmed that the archaeal diversity was adequately covered (Additional file 1, Fig. S1a and b). The non-chimeric sequences were clustered into 2,583 ASVs. 
Table 1

Summary of raw and filtered sequences

\begin{tabular}{|c|c|c|c|c|}
\hline Host species & Region & Raw sequences & Filtered sequences & $\%$ Filtered sequences \\
\hline \multirow[t]{2}{*}{ Cattle } & North & 4551909 & 445492 & 9.79 \\
\hline & South & 2445566 & 363215 & 14.85 \\
\hline Total & & 6997475 & 808707 & 11.56 \\
\hline \multirow[t]{2}{*}{ Buffaloes } & North & 2984245 & 457068 & 15.32 \\
\hline & South & 1331570 & 287090 & 21.56 \\
\hline Total & & 4315815 & 744158 & 17.24 \\
\hline Total (Cattle + Buffalo) & & 11313290 & 1552865 & 13.73 \\
\hline
\end{tabular}

The current study presents the findings on the comparative analysis of methanogen diversity in cattle and buffaloes at the country as well as intra- and inter-region level.

\section{Country Level Diversity}

Results from the study confirmed that the methanogens belonging to Methanobacteriales, Methanomicrobiales and Methanomassiliicoccales orders were present in the rumen of cattle and buffaloes (Fig. 2). Among the Methanobacteriales, Methanobrevibacter (Mbr.) constituted largest fraction of the rumen archaeal community in both cattle $(\nabla 55 \%)$ and buffaloes ( $\nabla 62 \%)$, although the difference in its abundance between the two bovine species was statistically insignificant. Mbr. gottschalkii clade was the most prominent species in both cattle and buffaloes, however, their abundance was significantly higher $(P=0.015)$ in cattle. Methylotrophic methanogens (Methanomassiliicoccales) represented approximately $2-4 \%$ of the total rumen archaeal community; nevertheless, they were significantly different between cattle and buffaloes (Additional file 2). Particularly, the abundances of Group 8 and Group 12 genera were significantly higher in cattle $(P=0.0011$ and 0.0002 , respectively), whereas, the distribution of Methanomicrobium (Mm.) Methanobacterium (Mb.) was higher in buffaloes, but not statistically significant. Among the Methanobrevibacter species, Mbr. wolinii was exclusively identified in the cattle. As expected, a large fraction of the rumen archaea (20-30\%) remained unclassified in the current study.

\section{Inter-region Diversity}

Similar to the country level, Methanobrevibacter was the most dominant genus in the cattle from both north and south regions (Fig. 3a and Additional file 3), although their distribution was statistically 
insignificant. However, Mbr. gottschalkii clade was significantly higher $(P<0.00016)$ in the cattle from the southern region. The diversity of methanogens affiliated to Methanobacterium, Methanomicrobium and Methanosphaera was not significantly different in cattle across the geographical regions. Moreover, the distribution of methylotrophic methanogen species, including Group $4(P=0.001)$, Group $8(P=0.011)$, Group $10(P=0.001)$ and Group $9(P<0.001)$ was higher in the cattle from the northern region (Additional file 2). Mbr. wolinii and Group $12 \mathrm{sp}$. ISO4 H5 methanogens were exclusively identified in cattle from the south and north regions, respectively (Fig. 3a).

Comparative analysis of methanogen diversity between the two geographical regions revealed a significantly higher abundance $(P=0.024)$ of Methanobacterium in the buffaloes from the southern region. Unlike in cattle, the Group 12 affiliated methanogens were identified in the buffaloes of both the regions (Fig. 3b and Additional file 3), but with a significantly higher $(P<0.001)$ abundance in the northern region (Additional file 2). The distribution of prominent rumen methanogen species, such as $M b r$. gottschalkii clade, Mbr. ruminantium clade, and $M b$. alkaliphilum was not significantly different between the two regions (Additional file 2). In contrast to cattle, the distribution of $\mathrm{Mm}$. mobile was significantly higher $(P=0.022)$ in buffaloes from the southern region. Similarly, the abundance of Group $4 \mathrm{sp}$. MpT1 was higher in the buffaloes of southern region $(P=0.001)$, whereas, that of Group $12 \mathrm{sp}$. ISO4 H5 was more in the northern region $(P=0.017)$.

\section{Intra-region Diversity}

Comparison of methanogen diversity between the bovine species within the geographical regions indicated a higher abundance of Methanobrevibacter $(P=0.0003)$ and Methanomicrobium $(P=0.0002)$ in buffaloes in the northern region. However, Group $8(P=0.003)$ and Group $9(P=0.009)$ were comparatively higher in cattle (Fig. $4 a$ and b, Additional file 2). Furthermore, at species level, the distribution of Methanosphaera sp. ISO3 F5, Group 4 MpT1, Group 8, Group $12 \mathrm{sp}$. ISOH5 and Group $9 \mathrm{sp}$. was higher in cattle than buffaloes (Fig. $4 a$ and b, Additional file 2). On the contrary, the prominent methanogen species, such as Mbr. gottschalkii clade and Mbr. ruminantium clade did not differ significantly between the two host species within the geographical regions.

Unlike in the north region, Group 4 was the only genus that showed significant differential abundance between cattle and buffaloes $(P=0.013)$ in southern region (Fig. 4b, Additional file 2). Furthermore, in the southern region, Mbr. gottschalkii clade had a higher abundance $(P=0.035)$ in cattle than in buffaloes, whereas, the abundance of Group $10 \mathrm{sp}$. was two times more in the buffaloes $(P=0.0047)$.

Methanogens, such as Mbr. wolinii and Group $9 \mathrm{sp}$. were exclusively present in the cattle; while Group 12 $s p$. ISO4 $\mathrm{H} 5$ was exclusive to buffaloes from the southern region. However, all the identified methanogen genera and species in the northern region were found in both cattle and buffaloes. The abundance of methanogens between the two regions and host species has been presented in Additional file 1, Figure S2 and S3, respectively. Additional file 4 and 5, respectively provide the number of sequences classified at genus and species level for the individual samples. 


\section{Core Microbiome Analysis}

Core microbiome analysis revealed that five genera were shared between cattle and buffaloes at a minimum detection threshold of $0.001 \%$. The prevalence of Group 4, Group 9 and Methanimicrococcus was observed only in cattle (Fig. 5a). Mbr. gottschalkii clade, Mbr. ruminantium clade and Mb. alkaliphilum were the most prevalent species in both cattle and buffaloes (Fig. 5a). Furthermore, the core microbiome analysis showed Methanobrevibacter and Methanobacterium to be prevalent in cattle and buffaloes across northern and southern regions (Fig. 5b-e). Interestingly, Methanomassiliicoccales related methanogens were not prevalent in south buffaloes (Fig. 5e), whereas, the prevalence of Methanosphaera was not observed specifically in the northern counterpart (Fig. 5d). At species level, Mb. alkaliphilum and $\mathrm{Mm}$. mobile were core microbiome members in both the bovine species and regions except in south cattle and south buffaloes, respectively.

\section{Diversity Index}

Alpha diversity for methanogen abundance assessed through Shannon index/ Pielou's eveness for country, inter-region and intra-region analysis has been presented in Fig. 6 . Shannon diversity index (Fig. 6a-c) revealed a significant difference in the methanogen abundance at country level $(P=0.014)$, inter-region $(P=0.002)$ and within the geographical region $(P=0.001)$. Similarly, the Pielou's evenness analysis (Additional file 1, Table S2) established a significant difference (Fig. 6d-f) in the methanogen diversity in bovine species between the northern and southern regions $(P=0.008)$ and across the bovine species between the two regions $(P=0.0005)$.

The beta-diversity analysis showed a distinct pattern for the clustering of samples from northern and southern regions on qualitative (Fig. 7a) and quantitative (Fig. 7b) distance matrices. Furthermore, the samples from the same species (cattle or buffalo) were more closely clustered.

\section{Network Analysis}

Network analysis (Fig. 8a-d) revealed a strong correlation across the different genera of the rumen methanogens. In the northern region (Fig. 8a), Group 9 and Group 4 were positively correlated $(r=0.93, P$ $=7.56 \mathrm{E}-06)$ in cattle; whereas, Group 9 showed a negative correlation with Methanobrevibacter $(r=-0.7, P$ $=0.010)$. Methanomicrobium and Group 8 were positively correlated $(r=0.96, P=1.66 \mathrm{E}-06)$ in buffaloes (Fig. 8b); however, Methanomicrobium and Methanobrevibacter were negatively correlated $(r=-0.93, P=$ 2.02E-05) within the northern region. A positive correlation between Methanimicrococcus and Group 4 ( $r$ $=0.95, P=4.41 \mathrm{E}-06)$ as well as Group 4 and Methanomicrobium $(r=0.87, P=5.83 \mathrm{E}-04)$ in cattle was evident in southern region (Fig. $8 \mathrm{c}$ and d). Furthermore, a negative correlation between Methanomicrobium and Methanobrevibacter was reported in buffaloes $(r=-0.94, P=5.68 \mathrm{E}-05)$. Heatmap also established a similar correlation among the methanogens between the two host species and regions (Fig. 8e). 


\section{Discussion}

Methane emission is one of the metabolic inefficiencies which takes away a considerable fraction of the feed energy. About $39.5 \mathrm{KJ}$ of the energy is wasted for each litre of enteric methane emission from the ruminants [37]. Microbiota-host relationship is complex and varies from synergistic to competitive [22]. The host species can affect the rumen archaeal community structure [23-26]. However, in spite of representing two major methane emitting species, the comparative diversity analysis of methanogens between cattle and buffaloes has not been extensively investigated. There is only one report where methanogen abundances between cattle and buffaloes were compared [38]. Community structure analysis from their study revealed a significantly higher abundance of Methanobrevibacter genus in Jersey cows than in water buffaloes. In addition, geographical location is known to influence on the methanogen community structure [9]. An analysis of 742 samples across 32 species and 35 countries confirmed that rumen archaea are less diverse than the bacteria [5]. The archaeal community was dominated by the members of Mbr. gottschalkii and Mbr. ruminantium clade along with Methanosphaera sp. and two Methanomassiliicoccales; they aggregately represent $~ 90 \%$ of the total archaea in the rumen. The abundances of these five dominant methanogens differed among the species [5]. Although India holds 13 and $53 \%$ of the global cattle and buffalo population, respectively [21]; they were not included in the study carried out by [5]. Therefore, the present study was aimed at comparing the rumen archaeal community structure between two host species (cattle \& buffaloes) and across geographical regions (north \& south) of India.

Our study revealed that the distribution of dominant methanogen genera i.e. Methanobrevibacter, Methanobacterium, Methanomicrobium and Methanosphaera was comparable between the two host species at the country level (Fig. 1; Additional file 2). Methanogens affiliated to Methanobrevibacter genus dominated and constituted $\sim 55-62 \%$ of the rumen archaeal community in cattle and buffaloes; however, its abundance was significantly higher $(P=0.0003)$ in buffaloes. These findings reaffirm the previous reports on the dominance of Methanobrevibacter methanogens in cattle [39, 40] and buffaloes [41, 42]. A meta-analysis of archaeal 16S rRNA sequences available in GenBank [43] supports our results for the similar distribution of Methanobrevibacter in cattle and buffaloes. The higher abundance of $\mathrm{Mbr}$. gottschalkii could be attributed to the higher proportion of straw in the diet of cattle. In India, cattle are poorly fed and comparatively inefficient milk producer than the buffaloes [44] which leads to the higher distribution of $M b r$. gottschalkii through higher $\mathrm{H}_{2}$ concentration and acetate/propionate (A/P) ratio [38, 45]. The difference in the distribution of Mbr. gottschalkii in the rumen of cattle and buffaloes was not equally dispersed in both the geographical regions and was more pronounced in the southern region. This could be due to the difference in climatic conditions as north India generally experiences cool and dry winters and hot summers; while the temperature is higher with humidity throughout the year in southern region. Further, the ruminants in southern region are mostly fed on the dry roughages, which may lead to the higher distribution of Mbr. gottschalkii. It has been reported that in addition to the geographical locations, feed restriction/under feeding [46] also leads to the higher abundance of Mbr. gottschalkii. In the present study, Mbr. wolinii was exclusively distributed in cattle. Earlier studies also confirmed the 
presence of Mbr. wolinii in the rumen of adult cattle [47-49]; while it remains unreported in the buffalo rumen [42,50, Malik et al., 2020, unpublished observations]. These findings suggest that the host species in conjunction with the geographical region and diet variation can affect the prevalence of some of the Methanobrevibacter species.

The abundance of $\mathrm{Mm}$. mobile did not differ between the two host species at the country level (Fig. 1; Additional file 2) as well as within the geographical regions (Fig. 3a-3b; Additional file 2). The distribution frequency of $\mathrm{Mm}$. mobile in cattle and buffaloes was in consonance with the global data sets $[5,12,49]$ but there is a disagreement with previous reports of unprecedented higher distribution of Methanomicrobium in cattle [51, 52] and buffaloes [53-55]. As these studies used PCR and cloning based approach; the primer bias could be one of the reason for the disagreement in Methanomicrobium abundance. A narrow difference in the distribution of $\mathrm{Mm}$. mobile in cattle and buffaloes established that the host species has little impact on its abundance, limited to $<10 \%$ of the total rumen archaea.

In the present study, Methanomassiliicoccales were significantly different between the host species and geographical regions (Fig. 1-3b; Additional file 2). Methanomassiliicoccales were previously reported in the rumen [26,56,57, Malik et al., 2020, unpublished observations] and their abundance frequency in this study are within the reported range of $<10 \%[24,57]$. Until recently, the Methanomassiliicoccales order was placed under the RCC (rumen cluster $\mathrm{C}$ ) and Methanoplasmatales [58-60]. Methanomassiliicoccales accomplish the reduction of methanol and methyl amines mediated by $\mathrm{H}_{2}$, as they lack the genes required for first six steps in hydrogenotrophic methanogenesis [61-63]. This study established that the distribution of Methanomassiliicoccaceae members, such as Group $8(P=0.001)$ and Group $12(P=$ 0.0002) was significantly higher in the cattle than in buffaloes. In an another study, Group 10, Group 11 and Group 12 were identified in New Zealand cattle and sheep [57]; whereas, in the current study, Group 4, Group 8, Group 9, Group 10 and Group 12 were identified in both the host species and geographical regions. However, the presence of Group 11 could not be confirmed in the rumen of cattle and buffaloes in the present study. Our results indicated that in spite of the small representation of Methanomassiliicoccales in the rumen archaeal community, they were more diversified in Indian ruminants than that of the New Zealand. A meta-analysis [43] strengthened our results on the higher abundance of Methanomassiliicoccales in cattle as compared to buffaloes. Though the methane producing ability of the Methanomassiliicoccales members has not yet been explored; their high relative abundance, i.e., up to $50 \%$ of the total rumen archaea $[24,57]$, is a clear indication of their possible involvement in rumen methanogenesis. Thus, the correlation between the abundance of Methanomassiliicoccales members and their methane producing ability warrants further investigation. In addition, non-availability of type strain hinders our understanding of the taxonomy, substrate requirement, and growth of Methanomassiliicoccales methanogens.

Findings from the current study established that the geographical region in conjunction with the host species has a higher influence on the abundance of Methanomassiliicoccales than the hydrogenotrophic archaea. The difference in the abundance of Methanomassiliicoccales between cattle and buffaloes could be due to the variable production of methylated substrate compounds in the rumen, which may be 
mediated through diet [64]. In addition, the differences in the environmental conditions influence the archaeal community structure [28]. A study from [27] in heifers concluded that the elevated temperatures along with high humidity significantly affects the rumen microbiota composition through the alteration in $A / P$ ratio. High environmental temperatures result in less rumen motility, which is known to increase the dry matter digestibility by prolonging the retention time of feeds in the rumen $[65,66]$. The climatic conditions of the two geographical regions; southern region (temp. $31^{\circ} \mathrm{C}$, humidity $78 \%$ ) is generally hotter than the northern counterpart in India (temp. $28.5^{\circ} \mathrm{C}$, humidity $59 \%$ ), may have contributed to the variable abundance of archaeal community structure in the current study. It is noteworthy that large size animals are more sensitive to the elevated temperature due to less dissipation of heat, which influences the abundance of rumen microbiota [27]. Hence, larger body size and weight of both the host species in the northern region may explain the higher abundance of Methanomassiliicoccales. However, the impact of increasing temperature and humidity on the distribution of various archaeal groups need further investigation.

Network analysis revealed both negative and positive correlations among different genera of methanogens in both the host and geographical region. Low interaction among the genera in buffaloes from south region was obviously due to the absence or relatively less abundance of many methanogens.

\section{Conclusions}

The core methanogens were similar between cattle and buffaloes, however, the overall archaeal community structure was influenced by the host species and geographical locations. Further, the archaeal community was dominated by Methanobrevibacter associated methanogens and the distribution of most prevalent Mbr. gottschalkii was significantly influenced by the host species in southern region. The distribution of Methanomassiliicoccales was significantly affected by the host species and geographical regions. Our study provides insights into the archaeal distribution in Indian cattle and buffaloes and established that the Methanomassiliicoccales due to their high variability can be one of the targets for achieving reduction in enteric methane emission. Further studies are warranted to determine the methane producing potential of Methanomassiliicoccales and explore its role in rumen methanogenesis.

\section{Abbreviations}

A/P- Acetate to propionate ratio

ASV- Amplicon sequence variants

$\mathrm{F}_{420^{-}}$8-hydroxy-5-deazaflavin coenzyme

GOI- Government of India

Mb- Methanobacterium 
Mbr- Methanobrevibacter

Mm- Methanomicrobium

NCBI- National Centre for Biotechnology Information

NGS- Next Generation Sequencing

OTU- Operational Taxonomic Unit

PERMANOVA- Permutational multivariate analysis of variance

ppb- parts per billion

RCC- Rumen cluster C

RIMDB- Rumen and Intestinal Methanogen Database

\section{Declarations}

\section{Ethical approval}

The study was approved by the Committee for the Purpose of Control and Supervision of Experiments on Animals (CPCSEA, 25/8/ 2016-CPCSEA part-I) to collect the rumen fluid samples. All the procedures were followed as per the Institute Animal Ethics Committee guidelines while collecting the samples.

\section{Consent for publication}

Not applicable

\section{Availability of data and material}

The datasets generated and/or analyzed during the current study are available in the NCBI Sequence Read Achieve (SRA; https://www.ncbi.nlm.nih.gov/sra) repository with accession numbers SAMN15083329-SAMN15083364 under BioProject PRJNA636770.

\section{Competing interests}

The authors declare that they have no competing interests

\section{Funding}

Authors thankfully acknowledge the financial support received from the Indian Council of Agricultural Research, New Delhi under outreach project on "Estimation of Methane Emission under Different Feeding systems and Development of Mitigation Strategies". 


\section{Authors' contributions}

PKM, APK, AD and RB designed the study. ST and TG collected the rumen fluid samples from cattle and buffaloes. ST, SV and TG performed DNA extraction, down processing and other laboratory work. ST, APK and VSK performed analysis of sequencing data. PKM supervised the findings of this work. RB arranged the financial support and provided logistics support for the study. All authors read and approved the final manuscript.

\section{Acknowledgements}

Support from Dr. A. K. Verma, Indian Veterinary Research Institute, Izatnagar;; Dr. M. Wadhwa, Guru Angad Dev Veterinary and Animal Science University, Ludhiana; Dr. P. R. Pandya, Anand Agricultural University, Anand and Dr. A. Natarajan, Tamil Nadu University of Veterinary and Animal Science, Namakkal for the rumen fluid sample collection is duly acknowledged. Critical inputs for bioinformatic analysis and interpretation from Mr. A.K. Bajpai are thankfully acknowledged. Authors also acknowledge the financial support received from Indian Council of Agricultural Research, New Delhi in conducting this study.

\section{References}

1. 10.1016/j.rser.2012.04.008

Yusuf RO, Noor ZZ, Abba AH, Hassan MAA, Din MFM. Methane emission by sectors: A comprehensive review of emission sources and mitigation methods. Renew Sustain Energy Rev [Internet]. Elsevier; 2012;16:5059-70. Available from: http://dx.doi.org/10.1016/j.rser.2012.04.008.

2. Saunois M, Jackson RB, Bousquet P, Poulter B, Canadell JG. The growing role of methane in anthropogenic climate change. Environ Res Lett. IOP Publishing; 2016;11.

3. Bhatta R, Malik PK, Kolte AP, Suresh KP. Assessment of enteric methane emission from Indian livestock: a new approach. In: Sejian V, Isloor S, Rahman SA, Bhatta R, editors. 7th Pan Commonw Vet Conf. Bengaluru: Commonwealth Veterinary Association(Asia); 2019. pp. 101-3.

4. Yanagita K, Kamagata Y, Kawaharasaki M, Suzuki T, Nakamura Y, Minato H. Phylogenetic analysis of methanogens in sheep rumen ecosystem and detection of methanomicrobium mobile by fluorescence in situ hybridization. Biosci. Biotechnol. Biochem. 2000. p. 1737-42.

5. Henderson G, Cox F, Ganesh S, Jonker A, Young W, Janssen PH, et al. Rumen microbial community composition varies with diet and host, but a core microbiome is found across a wide geographical range. Sci Rep. 2015;5.

6. Malik PK, Bhatta R, Soren NM, Sejian V, Mech A, Prasad KS, et al Feed-based approaches in enteric methane amelioration. In: Malik PK, Bhatta R, Takahashi J, Kohn RA, Prasad CS, editors. Livest Prod Clim Chang. CABl; 2015. p. 336-59.

7. Gagen EJ, Denman SE, McSweeney CS. Acetogenesis as an alternative to methanogenesis in rumen. In: Malik PK, Bhatta R, Takahashi J, Kohn RA, Prasad CS, editors. Livest Prod Clim Chang. CABI; 2015. p. 292-303. 
8. Balch WE, Fox GE, Magrum LJ, Woese CR, Wolfe RS. Methanogens: reevaluation of a unique biological group. Microbiol Rev. 1979;43:260-96.

9. Hook SE, Wright ADG, McBride BW. Methanogens. Methane producers of the rumen and mitigation strategies. Archaea. 2010;2010:50-60.

10. Greening C, Ahmed FH, Mohamed AE, Lee BM, Pandey G, Warden AC, et al. Redox reactions Redox reactions. 2016;80:451-93.

11. Holmes DE, Smith JA. Biologically Produced Methane as a Renewable Energy Source. Adv Appl Microbiol Elsevier Ltd. 2016;97:1-61.

12. Janssen PH, Kirs M. Structure of the archaeal community of the rumen. Appl Environ Microbiol. 2008;74:3619-25.

13. Kim CC. Identification of rumen methanogens, characterization of substrate requirements and measurement of hydrogen thresholds. 2012;1-141.

14. Wright ADG, Williams AJ, Winder B, Christophersen CT, Rodgers SL, Smith KD. Molecular Diversity of Rumen Methanogens from Sheep in Western Australia. Appl Environ Microbiol. 2004;70:1263-70.

15. Yu Z, García-González R, Schanbacher FL, Morrison M. Evaluations of different hypervariable regions of archaeal 16S rRNA genes in profiling of methanogens by Archaea-specific PCR and denaturing gradient gel electrophoresis. Appl Environ Microbiol. 2008;74:889-93.

16. Creevey CJ, Kelly WJ, Henderson G, Leahy SC. Determining the culturability of the rumen bacterial microbiome. Microb Biotechnol. 2014;7:467-79.

17. 10.1016/j.mimet.2010.10.011

Kenters N, Henderson G, Jeyanathan J, Kittelmann S, Janssen PH. Isolation of previously uncultured rumen bacteria by dilution to extinction using a new liquid culture medium. $\mathrm{J}$ Microbiol Methods [Internet]. Elsevier B.V.; 2011;84:52-60. Available from: http://dx.doi.org/10.1016/j.mimet.2010.10.011.

18. Leahy SC, Kelly WJ, Altermann E, Ronimus RS, Yeoman CJ, Pacheco DM, et al. The Genome Sequence of the Rumen Methanogen Methanobrevibacter ruminantium Reveals New Possibilities for Controlling Ruminant Methane Emissions. PLoS One. 2010;5.

19. Morgavi D, Kelly W, Janssen P, Attwood G, Morgavi D, Kelly W, et al. Rumen microbial (meta) genomics and its application to ruminant production To cite this version: HAL Id : hal-02647694 Rumen microbial ( meta ) genomics and its application to ruminant production. 2013.

20. Snelling TJ, Genç B, McKain N, Watson M, Waters SM, Creevey CJ, et al. Diversity and community composition of methanogenic archaea in the rumen of scottish upland sheep assessed by different methods. PLoS One. 2014;9.

21. Government of India [Internet]. 20th Livest. census provisional key results. Dep. Anim. Husb. Dairying, Minist. Fish. Anim. Husb. \{\\&\} Dairying, Govt. India. 2019. Available from: http://www.dahd.nic.in/division/provisional-key-results-20th-livestock-census.

22. Hungate RE. SYMPOSIUM. Microbes Nutr importance Aliment tract. Proceedings of the Niutrition Society; 1984 . p. 1-11. 
23. Zhou M, Hernandez-Sanabria E, Luo Guan L. Characterization of variation in rumen methanogenic communities under different dietary and host feed efficiency conditions, as determined by PCRdenaturing gradient gel electrophoresis analysis. Appl Environ Microbiol. 2010;76:3776-86.

24. St-Pierre B, Wright ADG. Diversity of gut methanogens in herbivorous animals. Animal. 2013;7:4956.

25. Huang XD, Tan HY, Long R, Liang JB, Wright ADG. Comparison of methanogen diversity of yak (Bos grunniens) and cattle (Bos taurus) from the Qinghai-Tibetan plateau, China. BMC Microbiol. 2012;12.

26. Huang XD, Martinez-Fernandez G, Padmanabha J, Long R, Denman SE, McSweeney CS. Methanogen Diversity in Indigenous and Introduced Ruminant Species on the Tibetan Plateau. Archaea. 2016;2016.

27. Tajima K, Nonaka I, Higuchi K, Takusari N, Kurihara M, Takenaka A, et al. Influence of high temperature and humidity on rumen bacterial diversity in Holstein heifers. Anaerobe. 2007;13:57-64.

28. von Keyserlingk GE, Mathison GW. The effect of ruminal escape protein and ambient temperature on the efficiency of utilization of metabolizable energy by lambs. J Anim Sci. 1993;71:2206-17.

29. Yu Z, Morrison M. Improved extraction of PCR-quality community DNA from digesta and fecal samples. Biotechniques. 2004;36:808-12.

30. Bolyen E, Rideout JR, Dillon MR, Bokulich NA, Abnet CC, Al-Ghalith GA, et al. Reproducible, interactive, scalable and extensible microbiome data science using QIIME 2. Nat Biotechnol. 2019;37:852-7.

31. Seedorf H, Kittelmann S, Henderson G, Janssen PH. RIM-DB: A taxonomic framework for community structure analysis of methanogenic archaea fromthe rumen and other intestinal environments.

PeerJ. 2014;2014.

32. Dhariwal A, Chong J, Habib S, King IL, Agellon LB, Xia J. MicrobiomeAnalyst: A web-based tool for comprehensive statistical, visual and meta-analysis of microbiome data. Nucleic Acids Res. 2017;45:W180-8.

33. Paulson JN, Colin Stine O, Bravo HC, Pop M. Differential abundance analysis for microbial markergene surveys. Nat Methods. 2013;10:1200-2.

34. Paul Shannon 1

Paul Shannon 1. AM 1, 00, 2 Nitin, Baliga S, 1 Jonathan T, Wang, 2 Daniel Ramage 2, Nada Amin 2,

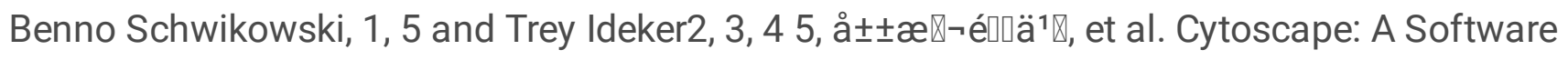
Environment for Integrated Models. Genome Res [Internet]. 2003;13:2498-504. Available from: http://ci.nii.ac.jp/naid/110001910481/.

35. Metsalu T, Vilo J. ClustVis. A web tool for visualizing clustering of multivariate data using Principal Component Analysis and heatmap. Nucleic Acids Res. 2015;43:W566-70.

36. Lahti L, Shetty S. Microbiome@GitHub [Internet]. [cited 2020 Jul 31]. Available from: http://microbiome.github.io/.

37. Guan H, Wittenberg KM, Ominski KH, Krause DO. Efficacy of ionophores in cattle diets for mitigation of enteric methane. J Anim Sci. 2006;84:1896-906. 
38. Iqbal MW, Zhang Q, Yang Y, Li L, Zou C, Huang C, et al. Comparative study of rumen fermentation and microbial community differences between water buffalo and Jersey cows under similar feeding conditions. J Appl Anim Res. 2018;46:740-8.

39. 10.1016/j.gene.2013.04.002

Sirohi SK, Chaudhary PP, Singh N, Singh D, Puniya AK. The 16S rRNA and mcrA gene based comparative diversity of methanogens in cattle fed on high fibre based diet. Gene [Internet]. Elsevier B.V.; 2013;523:161-6. Available from: http://dx.doi.org/10.1016/j.gene.2013.04.002.

40. Parmar NR, Pandit PD, Purohit HJ, Nirmal Kumar JI, Joshi CG. Influence of Diet Composition on Cattle Rumen Methanogenesis: A Comparative Metagenomic Analysis in Indian and Exotic Cattle. Indian J Microbiol. 2017;57:226-34.

41. Franzolin R, St-Pierre B, Northwood K, Wright A-DG. Analysis of Rumen Methanogen Diversity in Water Buffaloes (Bubalus bubalis) Under. Ecology [Internet]. 2012;64:131-9. Available from: https://www.jstor.org/stable/41489794?seq=1\&cid=pdf-reference\#references_tab_contents.

42. Kumar S, Dagar SS, Agrawal RK, Puniya AK. Comparative diversity analysis of ruminal methanogens in Murrah buffaloes (Bubalus bubalis) in four states of North India. Anaerobe Elsevier Ltd. 2018;52:59-63.

43. Paul SS, Dey A, Baro D, Punia BS. Comparative community structure of archaea in rumen of buffaloes and cattle. J Sci Food Agric. 2017;97:3284-93.

44. Indian cow, may your yield increase. The Hindu BusinessLine [Internet]. Bangalore; 2014 Mar 26; Available from: https://www.thehindubusinessline.com/opinion/indian-cow-may-your-yieldincrease/article22995029.ece.

45. McCabe MS, Cormican P, Keogh K, O’Connor A, O’Hara E, Palladino RA, et al. Illumina MiSeq phylogenetic amplicon sequencing shows a large reduction of an uncharacterised succinivibrionaceae and an increase of the Methanobrevibacter gottschalkii clade in feed restricted cattle. PLoS One. 2015;10:1-25.

46. Shi W, Moon CD, Leahy SC, Kang D, Froula J, Kittelmann S, et al. Methane yield phenotypes linked to differential gene expression in the sheep rumen microbiome Running title: Deep sequencing of sheep rumen microbiome with $\mathrm{CH} 4$ traits. 2014;1517-25.

47. Skillman LC, Evans PN, Naylor GE, Morvan B, Jarvis GN, Joblin KN. 16S ribosomal DNA-directed PCR primers for ruminal methanogens and identification of methanogens colonising young lambs. Anaerobe. 2004;10:277-85.

48. Wright ADG, Ma X, Obispo NE. Methanobrevibacter phylotypes are the dominant methanogens in sheep from Venezuela. Microb Ecol. 2008;56:390-4.

49. Zhou M, Hernandez-Sanabria E, Le LG. Assessment of the microbial ecology of ruminal methanogens in cattle with different feed efficiencies. Appl Environ Microbiol. 2009;75:6524-33.

50. 10.1016/j.anaerobe.2015.06.002

Paul SS, Deb SM, Dey A, Somvanshi SPS, Singh D, Rathore R, et al. 16S rDNA analysis of archaea indicates dominance of Methanobacterium and high abundance of Methanomassiliicoccaceae in 
rumen of Nili-Ravi buffalo. Anaerobe [Internet]. Elsevier Ltd; 2015;35:3-10. Available from: http://dx.doi.org/10.1016/j.anaerobe.2015.06.002.

51. Shin EC, Choi BR, Lim WJ, Hong SY, An CL, Cho KM, et al. Phylogenetic analysis of archaea in three fractions of cow rumen based on the 16S rDNA sequence. Anaerobe. 2004;10:313-9.

52. Tajima K, Aminov RI, Nagamine T, Matsui M, Nakamura M. YB. Diet dependent shifts in the bacterial population of the rumen revelaed by real time PCR. Appl Environ Microbiol [Internet]. 2001;67:276674. Available from: http://www.ceskatelevize.cz/ct24/veda/1954428-klimaticka-zmena-zasahnecesko-naplno-po-roce-2040-potvrdili-vedci-a-popsali-jeji.

53. Singh KM, Pandya PR, Parnerkar S, Tripathi AK, Rank DN, Kothari RK, et al. Molecular identification of methanogenic archaea from surti buffaloes (Bubalus bubalis), reveals more hydrogenotrophic methanogens phylotypes. Brazilian J Microbiol. 2011;42:132-9.

54. 10.1016/j.rvsc.2011.03.022

Singh KM, Tripathi AK, Pandya PR, Parnerkar S, Rank DN, Kothari RK, et al. Methanogen diversity in the rumen of Indian Surti buffalo (Bubalus bubalis), assessed by 16S rDNA analysis. Res Vet Sci [Internet]. Elsevier Ltd; 2012;92:451-5. Available from: http://dx.doi.org/10.1016/j.rvsc.2011.03.022.

55. Chaudhary PP, Sirohi SK. Dominance of Methanomicrobium phylotype in methanogen population present in Murrah buffaloes (Bubalus bubalis). Lett Appl Microbiol. 2009;49:274-7.

56. Poulsen M, Schwab C, Borg Jensen B, Engberg RM, Spang A, Canibe N, et al. Methylotrophic methanogenic Thermoplasmata implicated in reduced methane emissions from bovine rumen. Nat Commun. 2013;4.

57. Seedorf H, Kittelmann S, Janssen PH. Few highly abundant operational taxonomic units dominate within rumen methanogenic archaeal species in New Zealand sheep and cattle. Appl Environ Microbiol. 2015;81:986-95.

58. Dridi B, Raoult D, Drancourt M. Matrix-assisted laser desorption/ionization time-of-flight mass spectrometry identification of Archaea: Towards the universal identification of living organisms. Apmis. 2012;120:85-91.

59. lino T, Tamaki H, Tamazawa S, Ueno Y, Ohkuma M, Suzuki Kl, et al. Candidatus methanogranum caenicola: A novel methanogen from the anaerobic digested sludge, and proposal of Methanomassiliicoccaceae fam. nov. and Methanomassiliicoccales ord. nov., for a methanogenic lineage of the class Thermoplasmata. Microbes Environ. 2013;28:244-50.

60. Paul K, Nonoh JO, Mikulski L, Brune A. "Methanoplasmatales," thermoplasmatales-related archaea in termite guts and other environments, are the seventh order of methanogens. Appl Environ Microbiol. 2012;78:8245-53.

61. Jin W, Cheng Y, Zhu W. The community structure of Methanomassiliicoccales in the rumen of Chinese goats and its response to a high-grain diet. J Anim Sci Biotechnol Journal of Animal Science Biotechnology. 2017;8:1-10.

62. Lang K, Schuldes J, Klingl A, Poehlein A, Daniel R, Brune A. New mode of energy metabolism in the seventh order of methanogens as revealed by comparative genome analysis of "Candidatus 
Methanoplasma termitum..". Appl Environ Microbiol. 2015;81:1338-52.

63. Söllinger A, Schwab C, Weinmaier T, Loy A, Tveit AT, Schleper C, et al. Phylogenetic and genomic analysis of Methanomassiliicoccales in wetlands and animal intestinal tracts reveals clade-specific habitat. FEMS Microbiol Ecol. 2016;92:1-12.

64. Pol A, Demeyer DI. Fermentation of methanol in the sheep rumen. Appl Environ Microbiol. 1988;54:832-4.

65. 10.3168/jds.S0022-0302(90)78793-0

Martz FA, Payne CP, Matches AG, Belyea RL, Warren WP. Forage Intake, Ruminal Dry Matter Disappearance, and Ruminal Blood Volatile Fatty Acids for Steers in 18 and $32^{\circ} \mathrm{C}$ Temperatures. $\mathrm{J}$ Dairy Sci [Internet]. Elsevier; 1990;73:1280-7. Available from: http://dx.doi.org/10.3168/jds.S00220302(90)78793-0.

66. Christopherson RJ. PMK. Effect of the thermal environment on digestion in ruminants. Can J Anim Sci. 1983;63:477-96.

\section{Figures}

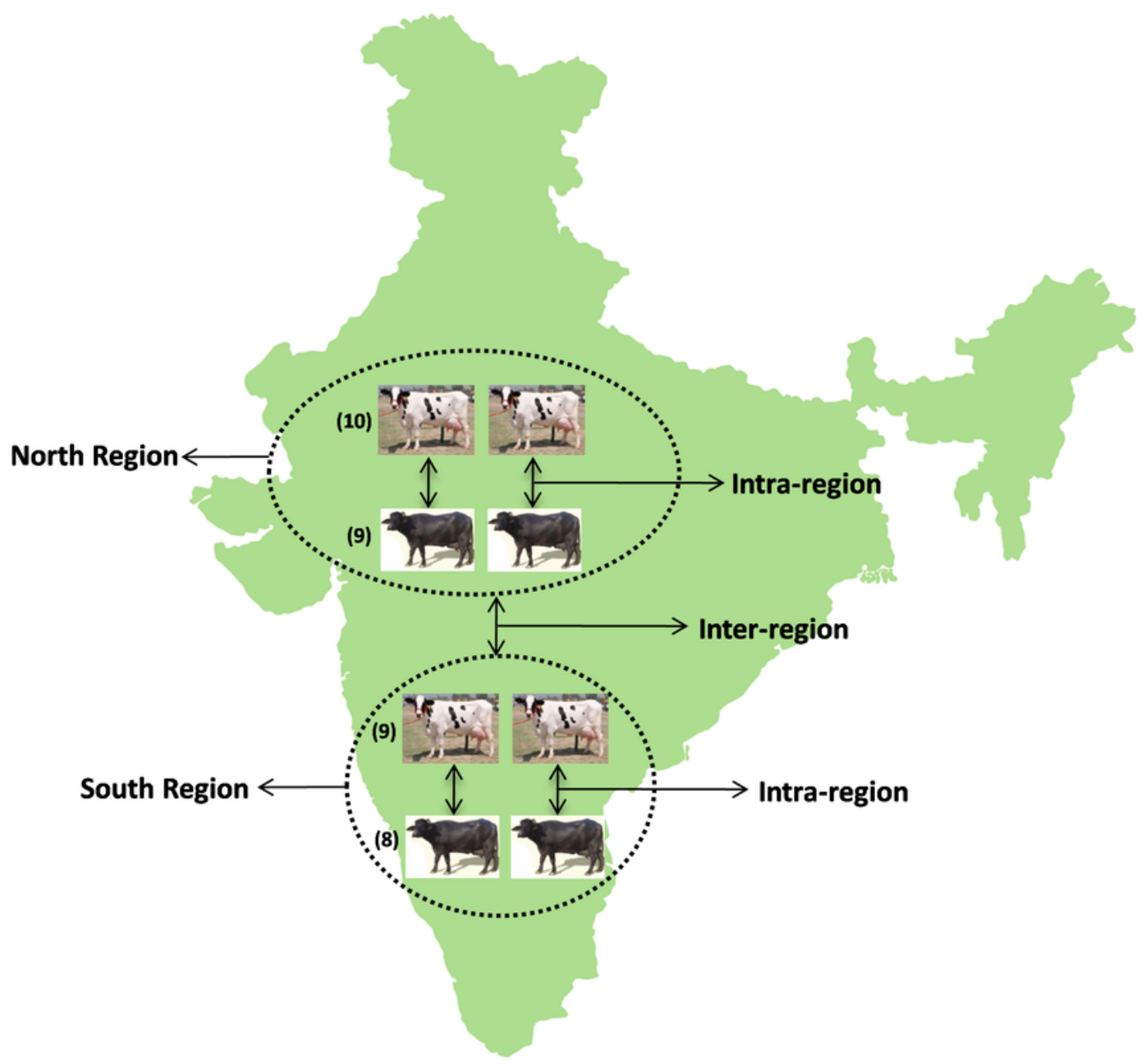


Figure 1

Species and region-wise rumen fluid collection

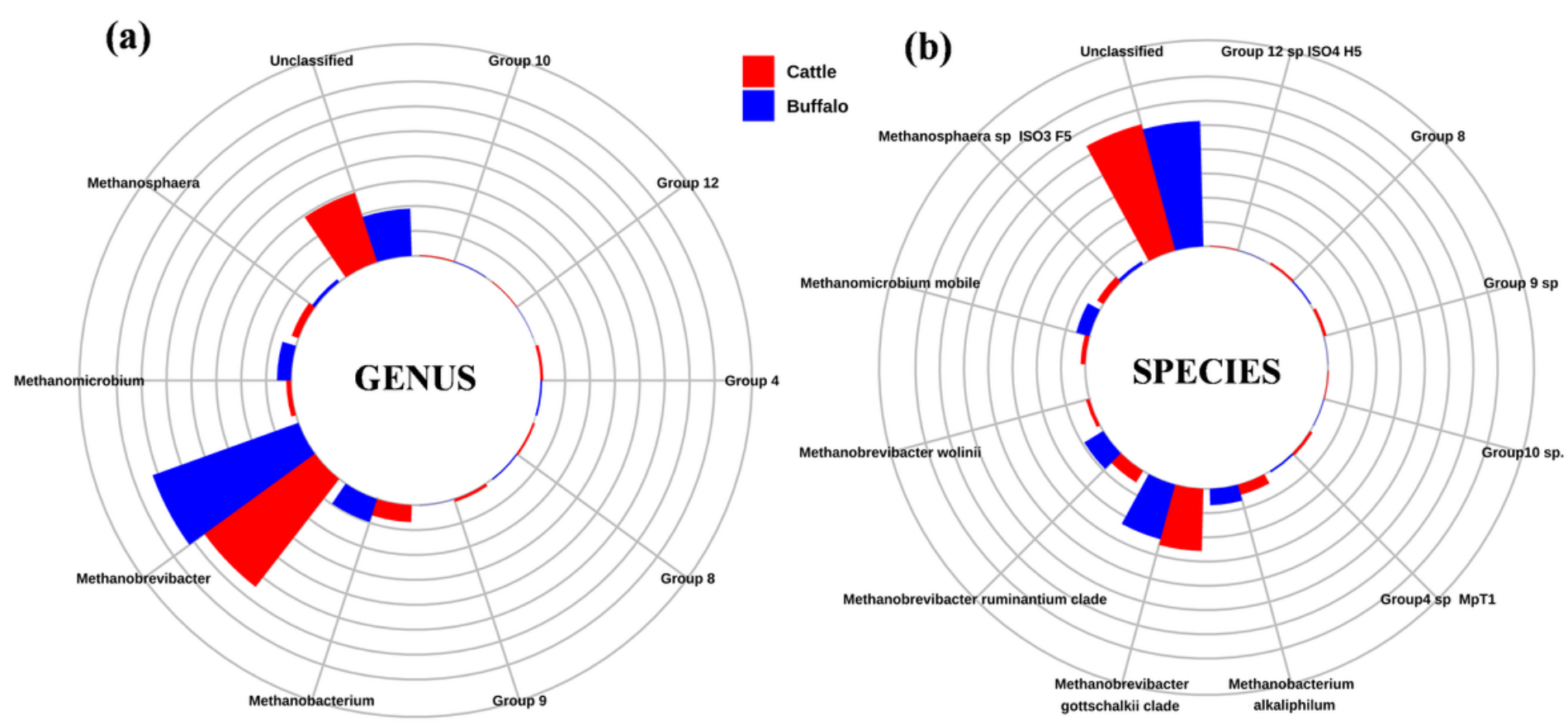

Figure 2

Comparative abundance of the rumen methanogens at (a) genus and (b) species level 

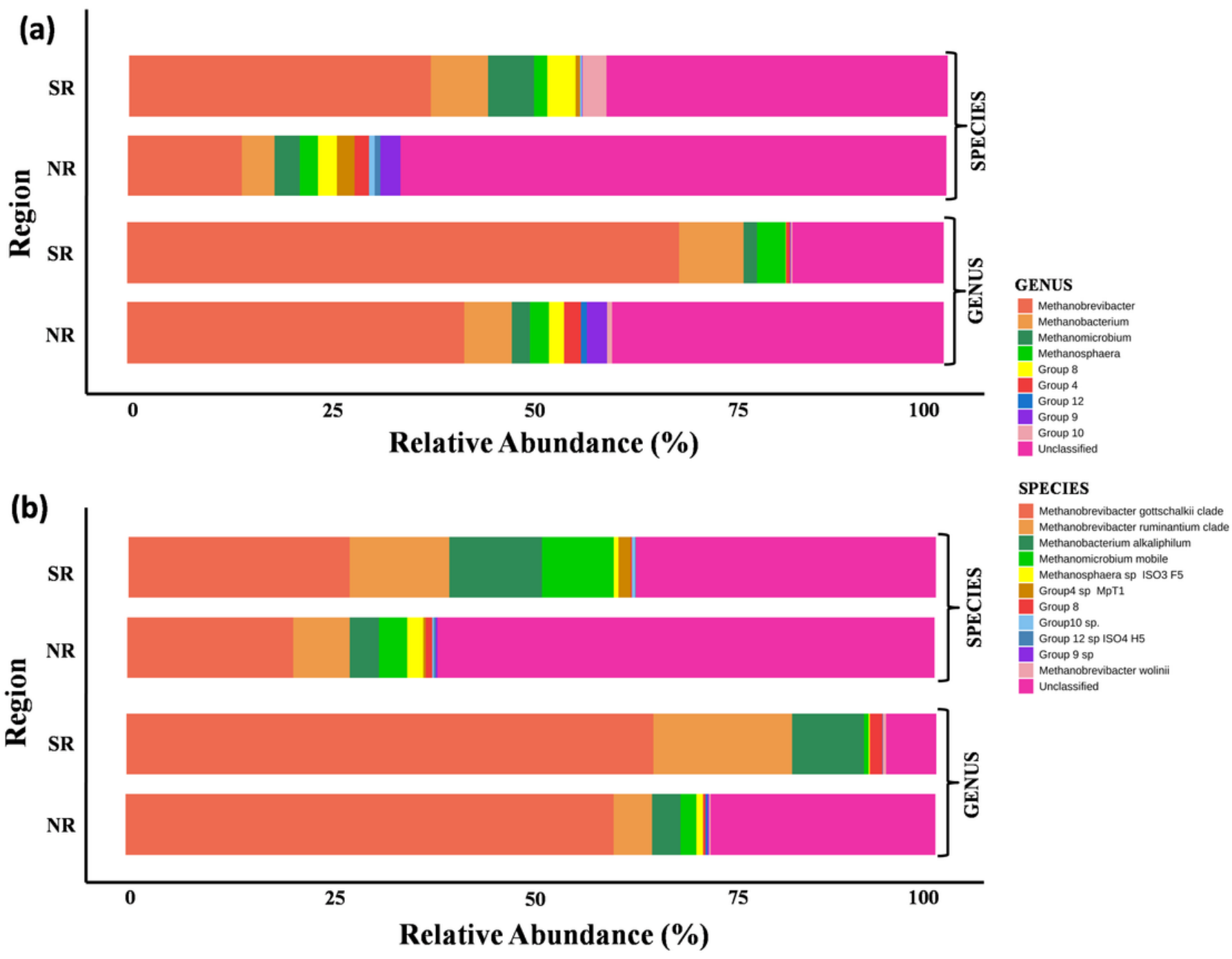

\section{Figure 3}

Inter-region abundance of the rumen methanogens in (a) cattle and (b) buffaloes. SR: Southern region; NR: Northern region. 

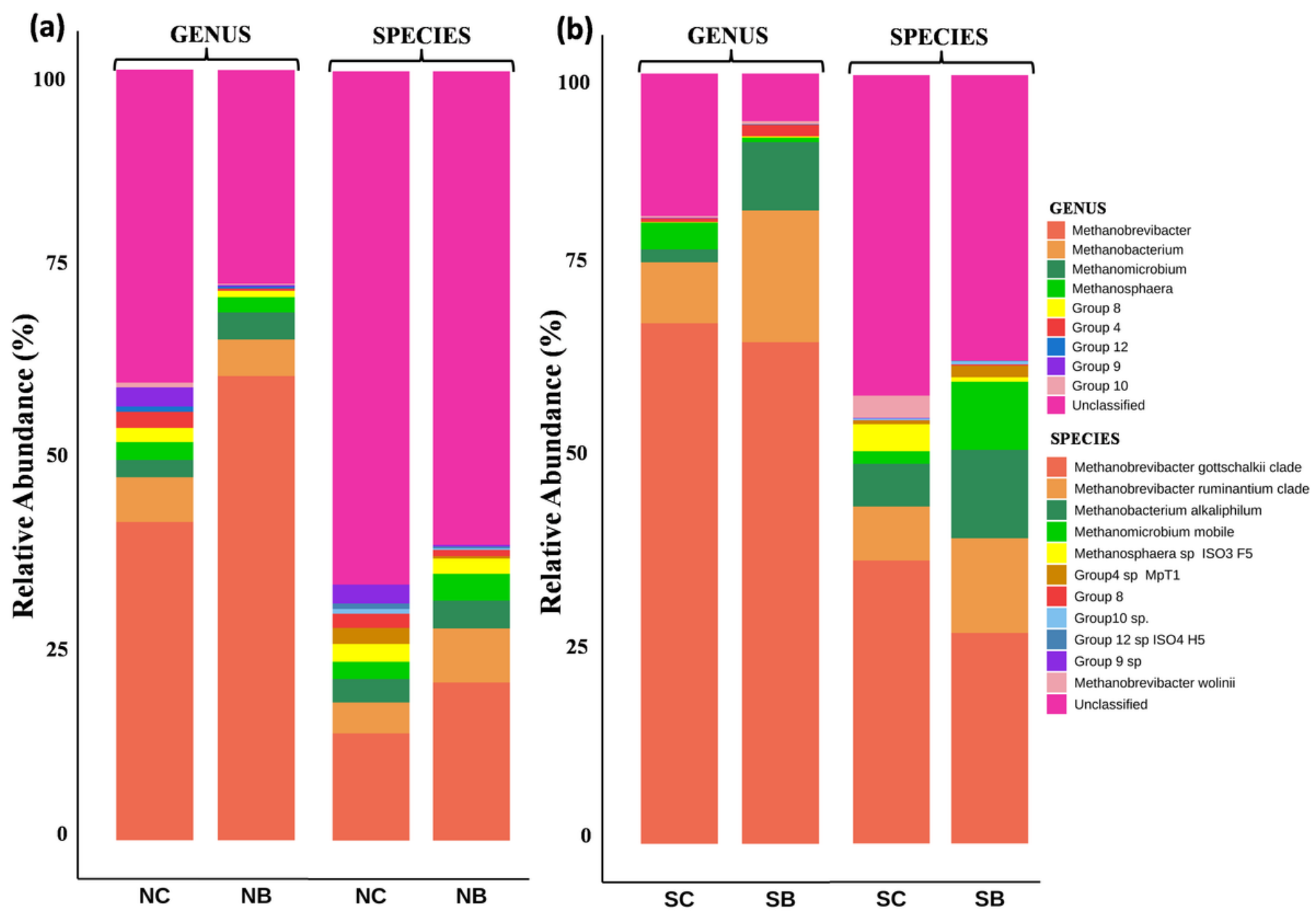

Figure 4

Relative abundance of rumen methanogens in (a) northern and (b) southern regions. NC: North cattle; NB: North buffalo; SC: South cattle; SB: South buffalo. 


\section{(a) Country Level}
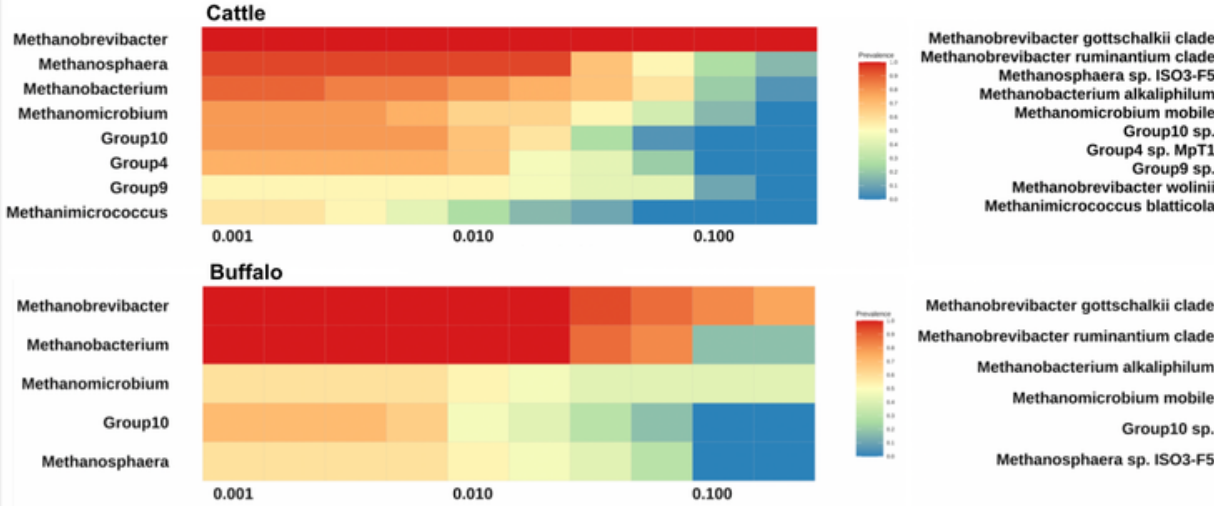

\section{(b) North Cattle}

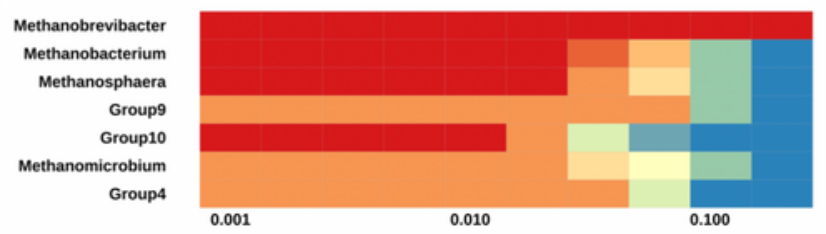

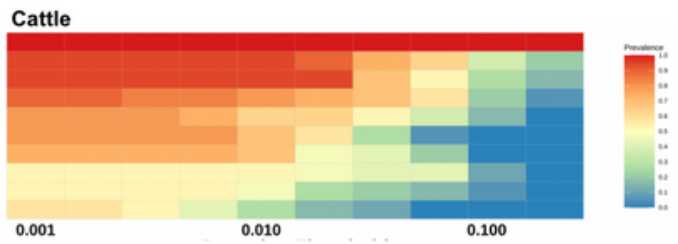

Buffalo

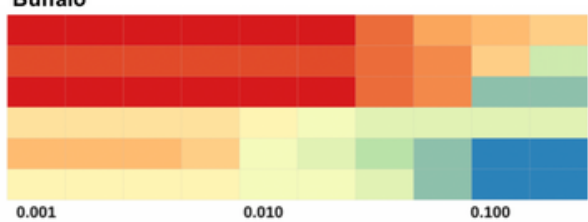

0.010

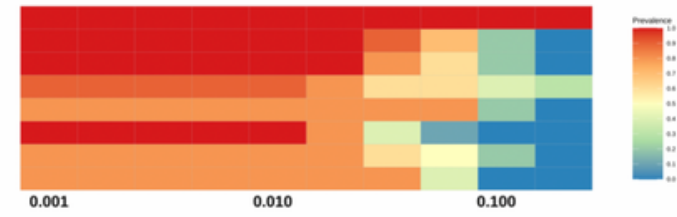

(c) South Cattle
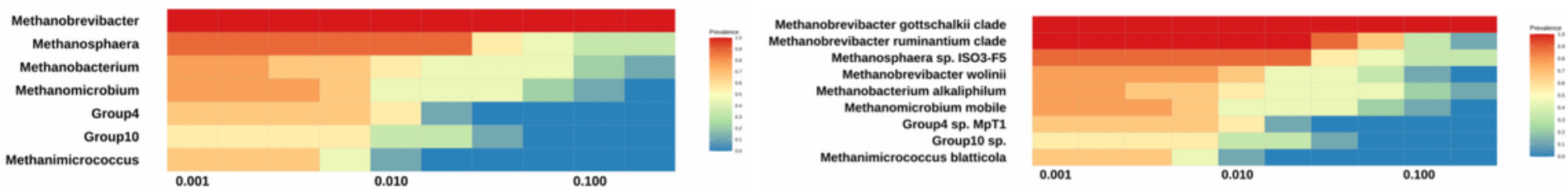

(d) North Buffalo
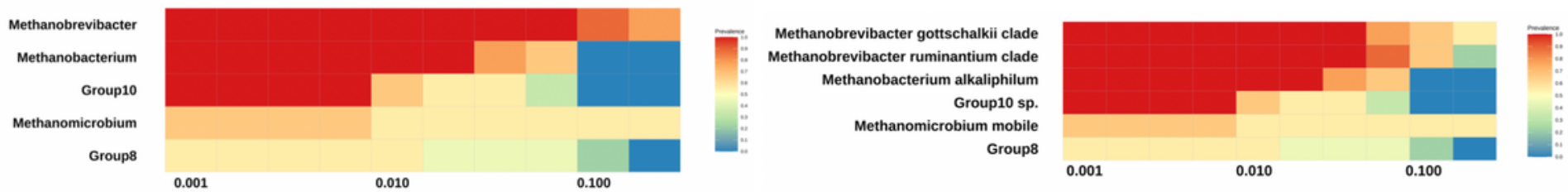

(e) South Buffalo
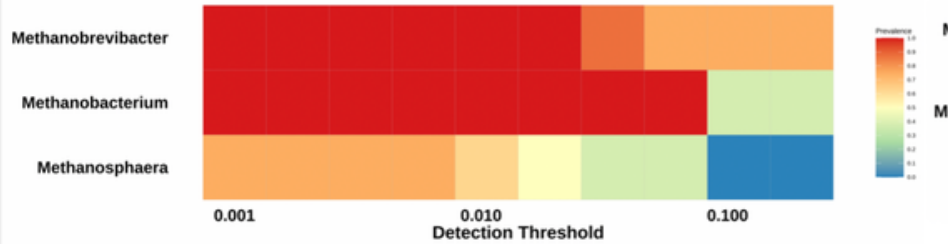

Methanobrevibacter gottschalkii clade Methanobacterium alkaliphilum Methanobrevibacter ruminantium clade Methanosphaera sp. ISO3-F5

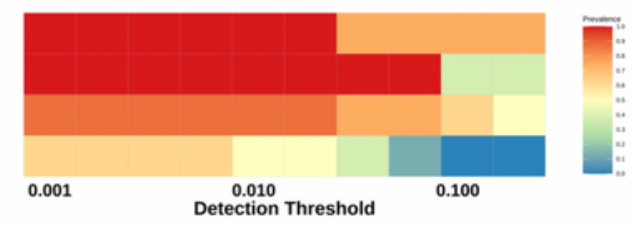

\section{Figure 5}

Core microbiome analysis at genus (left) and species (right) level. 

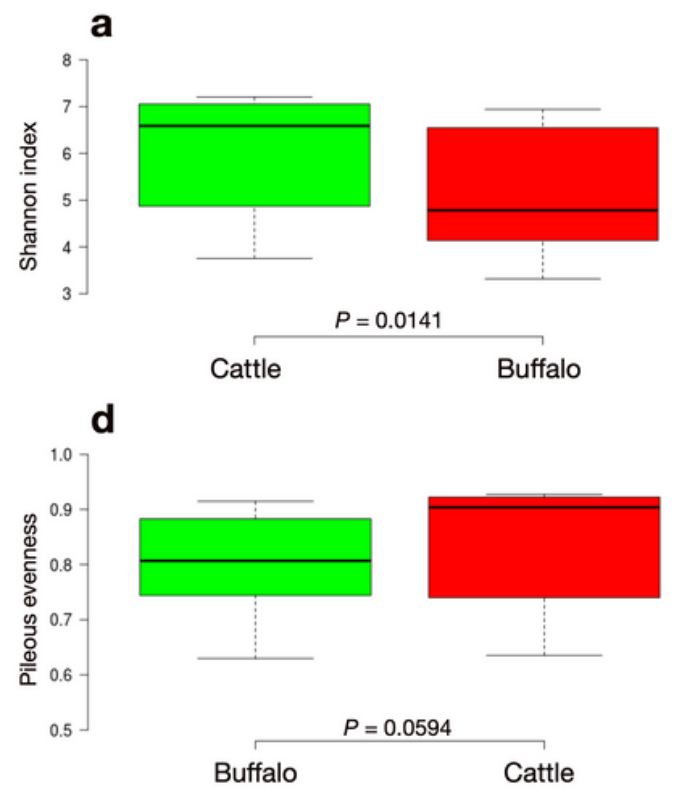

b
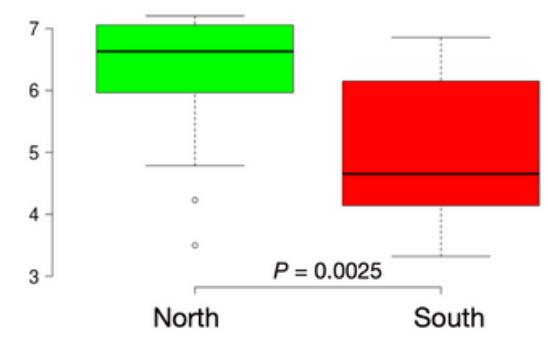

e

c

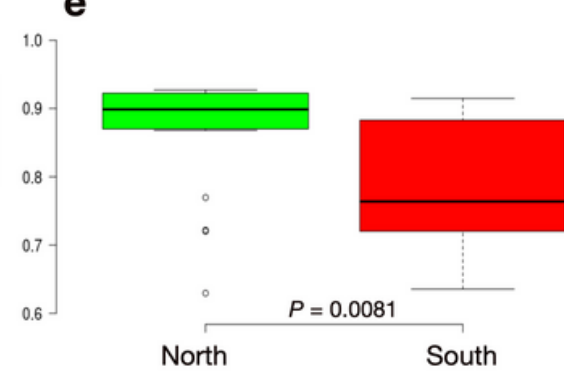

C

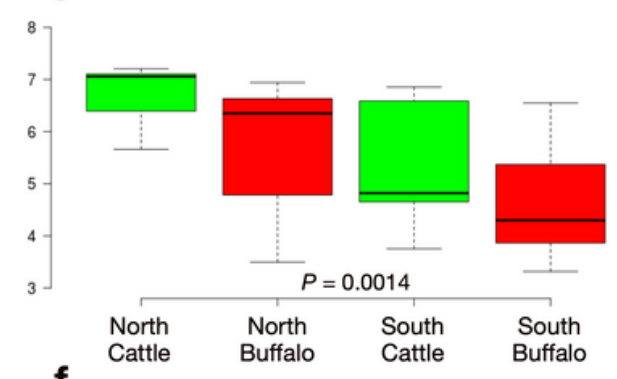

f

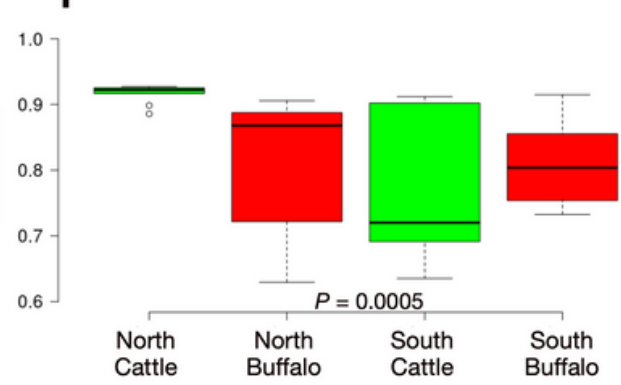

Figure 6

Alpha diversity indices. Shannon diversity index at country, region and intra-region levels is presented in a, $b$ and c, respectively. Figures $d$, e and $f$ represent the Pielou's eveness at country, region and intra-region levels, respectively.

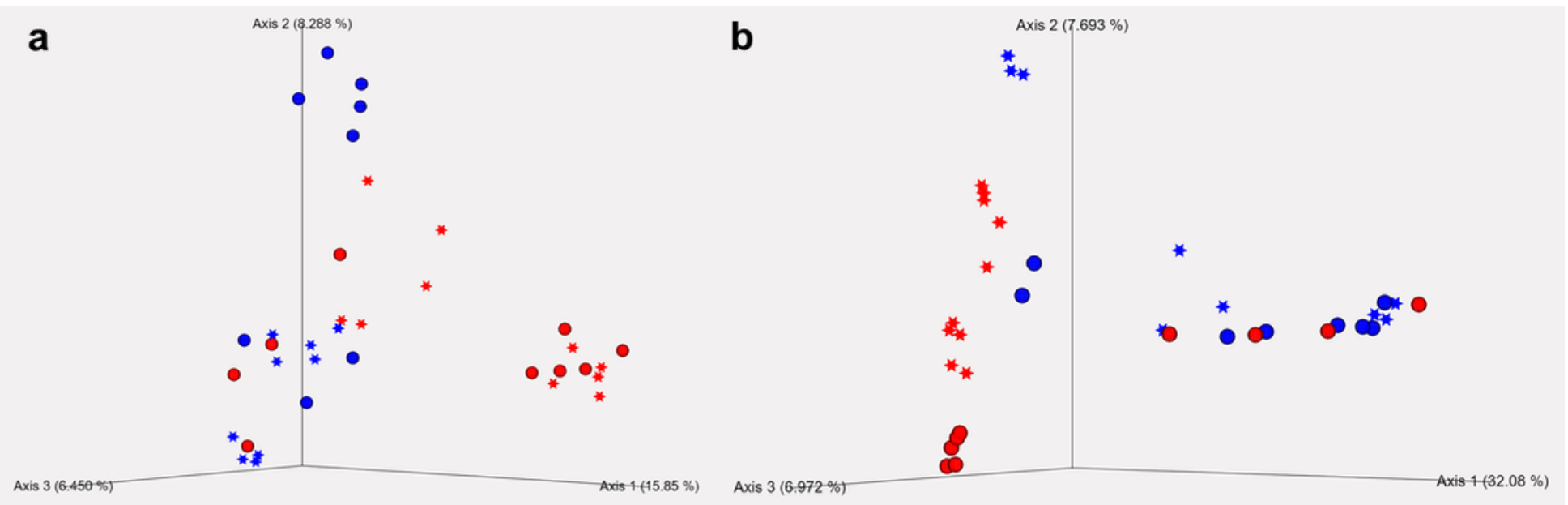

\section{Figure 7}

Beta diversity matrices. Principle coordinate analysis (PCoA) based on (a) unweighted UniFrac and (b) Bray-Curtis distance matrices. Color indicate the region and shape indicate the host species. red = north, blue = south; buffalo $=$ sphere, cattle $=$ star 


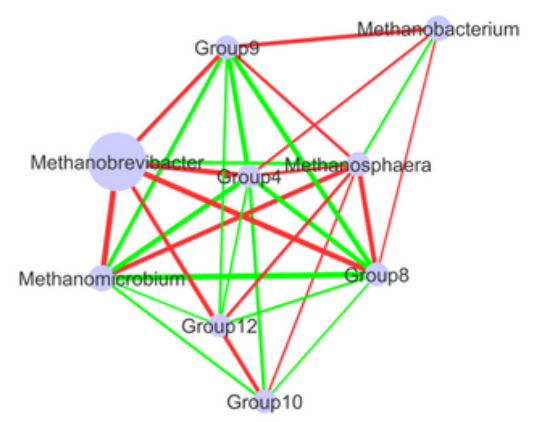

a

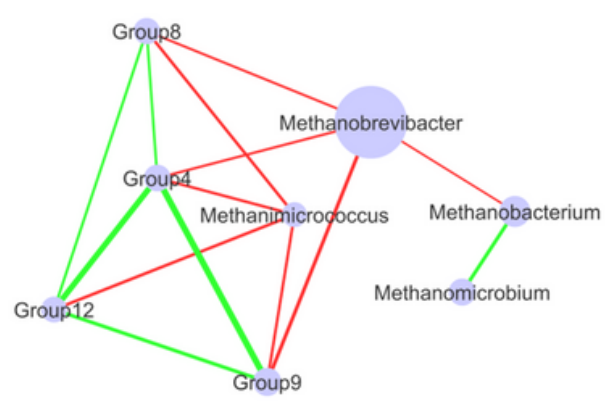

b

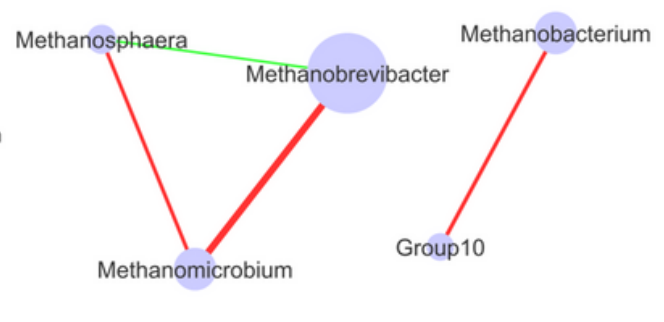

C

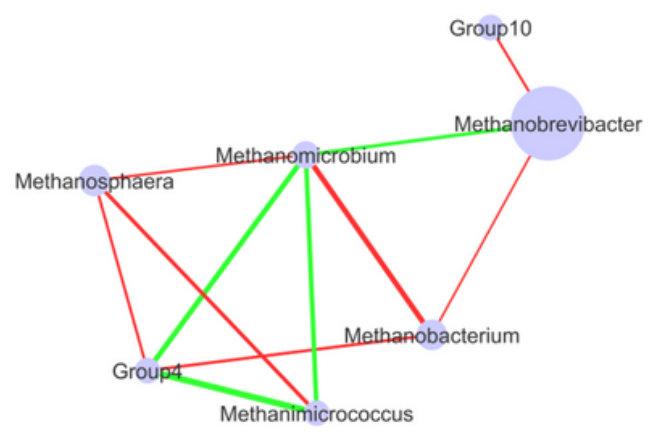

d

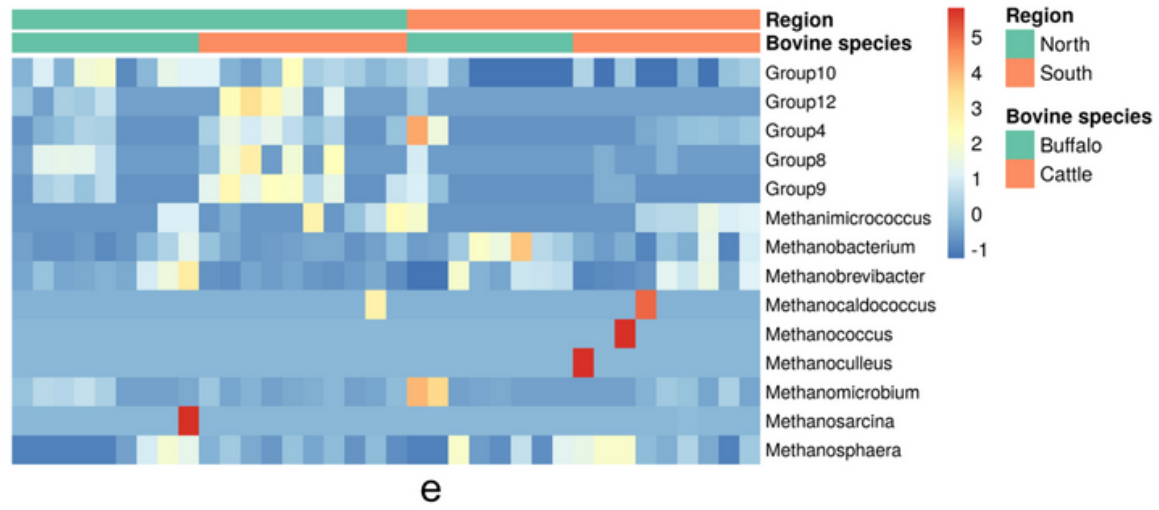

\section{Figure 8}

Network analysis of the methanogens representing the correlation across different genera. Correlation network of archaeal genera in (a) cattle and (b) buffalo from northern region. Correlation network of archaeal genera in (c) cattle and (d) buffalo from southern region. e, Heatmap of archaeal genera across all bovine samples. Copresence and mutual exclusion between the archaeal genera is represented with green and red edges, respectively. The edge thickness represents increasing correlation coefficient or weight (correlation coefficient threshold $=0.3$ ). Node size indicates abundance of archaeal genera.

\section{Supplementary Files}

This is a list of supplementary files associated with this preprint. Click to download.

- Graphicalabstract.png

- AdditionalFile5.xls

- AdditionalFile4.xls

- AdditionalFile3.xls

- AdditionalFile2.xls

- AdditionalFile1.doc 